\title{
Identification of novel genetic markers associated with the clinical phenotypes of systemic sclerosis through a genome wide association strategy
}

\author{
O Gorlova ${ }^{1 \dagger}$, J M Martin ${ }^{2 \dagger}$, B Rueda ${ }^{2 \dagger}$, BPC Koeleman ${ }^{3 \dagger}$, J Ying ${ }^{1}$, M Teruel $^{2}$, L M Diaz-Gallo ${ }^{2}$, J C Broen ${ }^{4}$, M C Vonk ${ }^{4}$, \\ C P Simeon ${ }^{5}$, B Z Alizadeh ${ }^{6}$, MJH Coenen ${ }^{7}$, A E Voskuyl ${ }^{8}$, A J Schuerwegh ${ }^{9}$, PLCM van Riel $^{4}$, M Vanthuyne $^{10}$, \\ R van 't Slot', A Italiaander', R A Ophoff', N Hunzelmann'11, V Fonollosa ${ }^{5}$, N Ortego-Centeno ${ }^{12}$, \\ M A González-Gay ${ }^{13}$, F J García-Hernández ${ }^{14}$, M F González-Escribano ${ }^{15}$, P Airo ${ }^{16}$, J van Laar ${ }^{17}$, J Worthington ${ }^{18}$,

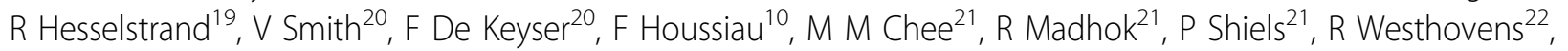

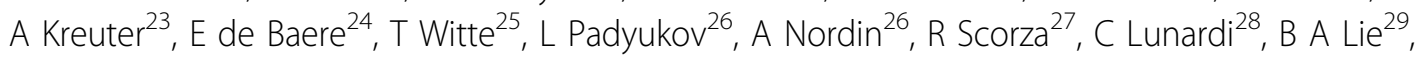 \\ A M Hoffmann-Vold ${ }^{30}$, P García de la Peña ${ }^{31}$, P Carreira ${ }^{13,32}$, J Varga ${ }^{33}$, M Hinchcliff ${ }^{33}$, A T Lee ${ }^{34}$, P Gourh ${ }^{35}$, \\ C I Amos ${ }^{1}$, G Riemekasten ${ }^{36}$, A Herrick ${ }^{18}$, L Beretta ${ }^{27}$, C Fonseca ${ }^{37}$, C P Denton ${ }^{37}$, P K Gregersen ${ }^{34}$, S Agarwal ${ }^{35}$,

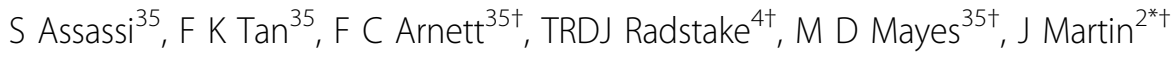 \\ From 5th European Workshop on Immune-Mediated Inflammatory Diseases \\ Sitges-Barcelona, Spain. 1-3 December 2010
}

\section{Purpose}

The aim of this study was to determine the genetic components contributing to the different systemic sclerosis (SSc) clinical sub-phenotypes of limited (lcSSc) and diffuse (dcSSc) cutaneous involvement, and with the most common SSc-specific autoantibodies, anticentromere (ACA) and anti-topoisomerase I (ATA) through a genome-wide association study (GWAS) and a large replication study stratified for these disease features.

\section{Methods}

In the discovery phase 2,296 SSc patients and 5,171 healthy controls were analyzed for genetic associations in lcSSc, dcSSc, ACA positive and ATA positive subgroups. The non-HLA SNPs associated with each subphenotype with a genomic control corrected $P$ value lower than $1 \times 10^{-5}$, not previously associated with SSc, were selected for replication in 9 independent cohorts from the US and Europe comprising an additional 3,175 SSc patients and 4,971 controls. In addition, meta-analyses including all patients and healthy controls were conducted for each subphenotype.

\footnotetext{
† Contributed equally

${ }^{2}$ Instituto de Parasitología y Biomedicina López-Neyra, Consejo Superior de Investigaciones Científicas, Granada, Spain

Full list of author information is available at the end of the article
}

\section{Results}

Three out of the 18 non-HLA SNPs selected for replication showed evidence of association. Meta-analysis including GWAS and replication cohorts showed a strong association of IRF8 $\mathrm{rs} 11642873$ polymorphism ( $\mathrm{P}=$ $\left.2.32 \times 10^{-12}, \mathrm{OR}=0.75\right)$ and a suggestive but consistent association among populations of GRB10 rs12540874 polymorphism $\left(\mathrm{P}=1.27 \times 10^{-6}, \mathrm{OR}=1.15\right)$ with the $\mathrm{lcSSc}$ subtype of the disease. Furthermore, significant association of SOX5 rs 11047102 polymorphism $\left(\mathrm{P}=1.39 \times 10^{-7}\right.$, OR = 1.36) with the ACA positive patients was detected. In the HLA region, specific patterns of SNPs associated with the ACA and ATA subgroups were observed, reflected by highly associated haplotype in the $H L A-D Q B 1$ locus with ACA $\left(P=1.79 \times 10^{-61}\right)$, and in the HLA-DPA1/B1 loci allelic combination with ATA $\left(P=4.57 \times 10^{-76}\right)$.

\section{Conclusions}

We have identified three new genes (IRF8, GRB10, and SOX5) associated with clinical manifestations of SSc, emphasizing the differential genetic component of each subphenotype of this disease. Within the HLA region, we have observed that $H L A-D Q B 1$ and $H L A-D P A 1 / B 1$ associations with SSc are likely confined to specific auto-antibody positive patients. 


\section{Author details}

${ }^{1}$ Dept. of Epidemiology, M.D. Anderson Cancer Center, Houston, Texas, USA. ${ }^{2}$ Instituto de Parasitología y Biomedicina López-Neyra, Consejo Superior de Investigaciones Científicas, Granada, Spain. ${ }^{3}$ Dept. of Medical Genetics, University Medical Center Utrecht, Utrecht, The Netherlands. ${ }^{4}$ Dept. of Rheumatology, Radboud University Nijmegen Medical Center, Nijmegen, The Netherlands. ${ }^{5}$ Servicio de Medicina Interna, Hospital Valle de Hebron, Barcelona, Spain. ${ }^{6}$ University Medical Centre Groningen, Dept. of Epidemiology, Groningen, The Netherlands. 'Dept. of Human Genetics, Radboud University Nijmegen Medical Center, Nijmegen, The Netherlands. ${ }^{8}$ Dept. of Rheumatology, Vrije Universiteit (VU) Medical Centre, Amsterdam, The Netherlands. 'Dept. of Rheumatology, University of Leiden, Nijmegen, The Netherlands. ${ }^{10}$ Cliniques Universitaires Saint-Luc, Université catholique de Louvain, Brussels, Belgium. " $D$ ept. of Dermatology, University of Cologne, Cologne, Germany. ${ }^{12}$ Servicio de Medicina Interna, Hospital Clínico Universitario, Granada, Spain. ${ }^{13}$ Servicio de Reumatología, Hospital Marqués de Valdecilla, Santander, Spain. ${ }^{14}$ Servicio de Medicina Interna, Hospital Virgen del Rocio, Sevilla, Spain. ${ }^{15}$ Servicio de Inmunología, Hospital Virgen del Rocío, Sevilla, Spain. ${ }^{16}$ Rheumatology Unit and Chair, Spedali Civili, Università degli Studi, Brescia, Italy. ${ }^{17}$ Institute of Cellular Medicine, Newcastle University, Newcastle Upon Tyne, UK. ${ }^{18}$ Dept. of Rheumatology and Epidemiology, University of Manchester, Manchester Academic Health Science Centre, Manchester, UK. ${ }^{19}$ Dept. of Clinical Sciences, Division of Rheumatology, Lund University, Lund, Sweden. ${ }^{20}$ Ghent University, Ghent, Belgium. ${ }^{21}$ Centre for Rheumatic Diseases, Glasgow Royal Infirmary Glasgow, UK. ${ }^{22}$ Katholieke Universiteit Leuven, Leuven, Belgium. ${ }^{23}$ Dept. of Dermatology, Josefs-Hospital, Ruhr University Bochum, Germany. ${ }^{24}$ Center for Medical Genetics, Ghent University Hospital, Ghent, Belgium. ${ }^{25}$ Hannover Medical School, Hannover, Germany. ${ }^{26}$ Center for Molecular Medicine, Karolinska Institutet, Stockholm, Sweden. ${ }^{27}$ Referral Center for Systemic Autoimmune Diseases, Fondazione IRCCS Ca' Granda Ospedale Maggiore Policlinico and University of Milan, Milan, Italy. ${ }^{28}$ Dept. of Clinical and Experimental Medicine, University Hospital, University of Verona, Italy. ${ }^{29}$ Institute of Immunology, Oslo University Hospital Rikshospitalet, Oslo, Norway. ${ }^{30}$ Dept. of Rheumatology, Rikshospitalet, Oslo University Hospital, Oslo, Norway. ${ }^{31}$ Servicio de Reumatología, Hospital Ramón y Cajal, Madrid, Spain. ${ }^{32}$ Hospital 12 de Octubre, Madrid, Spain. ${ }^{33}$ Northwestern University Feinberg School of Medicine, Chicago, Illinois, USA. ${ }^{34}$ Feinstein Institute of Medical Research, Manhasset, New York, USA. ${ }^{35}$ The University of Texas Health Science Center-Houston, Houston, Texas, USA. ${ }^{36}$ Dept. of Rheumatology and Clinical Immunology, Charité University Hospital, Berlin, Germany. ${ }^{37}$ Centre for Rheumatology, Royal Free and University College School, London, UK.

Published: 25 November 2010

doi:10.1186/1479-5876-8-S1-O1

Cite this article as: Gorlova et al:: Identification of novel genetic markers associated with the clinical phenotypes of systemic sclerosis through a genome wide association strategy. Journal of Translational Medicine 2010 8(Suppl 1):01.

\section{Submit your next manuscript to BioMed Central and take full advantage of:}

- Convenient online submission

- Thorough peer review

- No space constraints or color figure charges

- Immediate publication on acceptance

- Inclusion in PubMed, CAS, Scopus and Google Scholar

- Research which is freely available for redistribution

Submit your manuscript at www.biomedcentral.com/submit
Biomed Central 